\title{
APLICACIÓN DE LAS LMI AL DISEÑO DE CONTROLADORES ROBUSTOS
}

\section{APPLiCATION OF LMI TO THE DESIGN OF ROBUST CONTROLLERS}

\author{
Edwin Aguilar-Jaramillo ${ }^{1, *}$, Víctor Proaño-Rosero ${ }^{1}$
}

\section{Resumen}

El diseño de controladores precisos en presencia de incertidumbre significativa requiere el uso de controladores robustos. Una gran variedad de especificaciones y restricciones de diseño de sistemas de control pueden ser expresadas en términos de desigualdades matriciales lineales (LMI-Linear Matrix Inequalities), las mismas que son resueltas mediante algoritmos de optimización convexa. En este artículo se presenta el diseño de controladores robustos mediante LMI para sistemas típicos cuyos modelos son obtenidos de la literatura de control a los cuales se completa con la incertidumbre de parámetros. Los controladores diseñados utilizan la realimentación de estados del sistema.

Palabras clave: controlador, incertidumbre, robustez, realimentación.

\section{Abstract}

The design of precise controllers in presence of significant uncertainly requires the use of robust controllers. A variety of specifications and design constraints for control systems can be expressed in terms of Linear Matrix Inequalities (LMI), which are solved using Convex Optimization algorithms. This article presents the design of robust controllers using LMI for typical systems whose models are obtained from the control literature to which are complemented with parameter uncertainty. The designed controllers use system state feedback.

Keywords: controller, uncertainty, robust, feedback.

\footnotetext{
${ }_{1, *}$ Departamento de Eléctrica y Electrónica DEEE, Universidad de las Fuerzas Armadas ESPE. Sangolquí-Ecuador. Autor para correspondencia: eraguilar@espe.edu.ec. (1) http://orcid.org/0000-0003-0202-6071,

(iD) http://orcid.org/0000-0002-6404-1077
} 


\section{Introducción}

Este artículo está organizado en secciones. La sección 1 es la presente introducción. En la sección 2 se definen los principales conceptos que sustentan el procedimiento de diseño como son la optimización convexa, la definición de una LMI, los problemas matemáticos que se resuelven con las LMI y el concepto de estabilidad cuadrática de Lyapunov. La sección 3 presenta los problemas de control que se resuelven mediante la aplicación de las LMI y la teoría de estabilidad de Lyapunov que es el puente que permite pasar la formulación de espacio de estado de un sistema hacia una LMI. En la sección 4 se presenta la teoría para la formulación del problema de diseño de un controlador de realimentación de estados mediante LMI. En la sección 5 se presentan los casos de estudio que son el diseño de sendos controladores para un sistema doble integrador y para un levitador magnético. Posteriormente en la sección 6 se presentan los resultados y una breve discusión de los mismos, para finalizar se presentan las conclusiones en la sección 7 .

\section{Conceptos}

\subsection{Optimización convexa}

La optimización es la búsqueda de la mejor decisión dentro de un conjunto de posibles candidatos que cumplen una condición. El mejor candidato es aquel con el que se consigue el índice de desempeño óptimo. La optimización convexa se caracteriza por la existencia de un único mínimo global. Las LMI son funciones convexas y se resuelven normalmente por métodos conocidos como de puntos interiores.

\subsection{Definición de una LMI}

La idea básica del método LMI es formular un problema dado como un problema de optimización con objetivo lineal y restricciones definidas como desigualdades matriciales lineales.

Una desigualdad lineal matricial es una restricción convexa de la forma presentada en las ecuaciones 1 y simplificada en la ecuación 2 :

$$
\begin{gathered}
F(x)=F_{0}+x_{1} F_{1}+\cdots+x_{n} F_{n}>0 \\
F(x)=F_{0}+\sum_{i=1}^{N} x_{i} F_{i}>0
\end{gathered}
$$

Donde:

- $x=\left(x_{1} \ldots x_{n}\right)$ es un vector de escalares desconocidos (vector de variables).

- $F_{i}$ son matrices simétricas reales conocidas para $0 \leq \mathrm{i} \leq \mathrm{n}$.
- '<0' indica que todos los valores propios de $\mathrm{F}(\mathrm{x})$ son mayores que 0 .

La consecuencia importante de la convexidad es que a pesar de que la LMI no tiene una solución analítica en general, esta puede ser resuelta numéricamente con la garantía de encontrar una solución, si existe alguna.

El enfoque LMI es particularmente atractivo por las siguientes razones:

a) Se han desarrollado soluciones numéricas muy eficientes que habilitan resolver problemas para los cuales no existe una solución analítica conocida.

b) La formulación es muy adecuada para problemas con incertidumbre en los datos. La incertidumbre se describe mediante estructuras detalladas y fronteras para mediante un procedimiento sistemático formular un problema de optimización que conduce a una solución robusta. Esta característica es muy útil para una amplia gama de problemas de ingeniería donde se presentan errores en las variables medidas o errores de modelamiento.

c) Permite imponer varias especificaciones en el proceso de diseño para explorar los compromisos y analizar los límites de desempeño y factibilidad.

\subsection{Problemas LMI}

Los problemas que se resuelven por técnicas LMI y que son utilizados en este artículo son:

\subsubsection{Problema de factibilidad}

Se llama a la prueba de si existen o no soluciones de $\mathrm{x}$ tal que $\mathrm{F}(\mathrm{x})>0$. La LMI se llama no-factible en caso de no existir solución alguna.

\subsubsection{Problema de optimización}

Consiste en la minimización de una función que define un criterio de desempeño sujeta a restricciones de tipo LMI, como por ejemplo: Minimizar $\mathrm{c}^{\mathrm{T}} \mathrm{x}$, tal que $\mathrm{F}(\mathrm{x})>0$.

\subsection{Estabilidad cuadrática de Lyapunov}

La definición de la estabilidad cuadrática de Lyapunov da origen al estudio de las LMI. Considérese el sistema sin entradas dado por la ecuación 3 :

$$
\dot{x}=A x
$$

Se dice que el sistema es cuadráticamente estable si y solo si existe una función cuadrática positiva $\mathrm{V} \in \mathfrak{R}$ tal que se cumpla la ecuación 4 :

$$
V=x^{T} P x
$$


Y $\dot{V}$ sea negativa. La función V se conoce como función de Lyapunov y entrega como resultado un valor real para cada vector $\mathrm{x}$. La función queda definida completamente por la matriz $\mathrm{P}$ que se conoce como matriz de Lyapunov y que se constituye en una matriz simétrica a ser encontrada mediante una LMI, así: encontramos $\mathrm{P}$ tal que cumpla la ecuación 5:

$$
P=P^{T} ; P>0
$$

Teniendo en cuenta la condición planteada en la ecuación 6:

$$
A^{T} P+P A<0
$$

\section{Aplicación de las LMI al diseño de sistemas de control}

Una de las ventajas de la formulación de las LMI es la habilidad de combinar varias expresiones de diseño de una manera numéricamente tratable. Dentro del área de control existen innumerables tipos de problemas que pueden ser tratados mediante técnicas LMI. En este artículo se realiza la aplicación de las LMI al problema diseño de retroalimentación de estado para sistemas con incertidumbre de parámetros mediante una formulación politópica. Se utiliza la técnica de ubicación de polos en regiones LMI para satisfacer especificaciones del sistema en el dominio del tiempo y el cumplimiento de múltiples objetivos especificados en términos de normas $\mathrm{H}_{\infty}$ y $\mathrm{H}_{2}$ de funciones de transferencia obtenidas de la descripción del sistema. [1]. En esta sección se introducen los conceptos involucrados para resolver estos problemas.

\subsection{Modelación de la incertidumbre sistemas dinámicos}

Existen dos clases de incertidumbre:

\subsubsection{Incertidumbre dinámica}

Que consiste en componentes dinámicos omitidos en el modelo lineal o en variaciones en el comportamiento dinámico durante el funcionamiento del sistema. Por ejemplo, modos flexibles de alta frecuencia, variaciones de tiempo retardadas, etc.

\subsubsection{Incertidumbre de parámetros}

Dada por la estimación aproximada de los valores de parámetros físicos, o de las variaciones de estos parámetros durante el funcionamiento del sistema. Esta incertidumbre puede presentarse por ejemplo en el amortiguamiento de los coeficientes en sistemas mecánicos, coeficientes aerodinámicos de dispositivos de vuelo, capacitores e inductores en circuitos eléctricos, etc. [2]

\subsection{Planteamiento de sistemas con incertidumbre de parámetros}

Existen dos formas de plantear sistemas con incertidumbre: a través de modelos dependientes de parámetros afines y modelos politópicos. En el presente artículo se utiliza únicamente la formulación politópica de los sistemas.

Se llama un sistema politópico a un sistema lineal variante en el tiempo de la forma presentada en las ecuaciones 7,8 :

$$
\begin{aligned}
& \dot{x}=A(t) x+B(t) u \\
& y=C(t) x+D(t) u
\end{aligned}
$$

Cuya matriz del sistema $\mathrm{S}(\mathrm{t})$ se define en la ecuación 9 como:

$$
S(t)=\left[\begin{array}{ll}
A(t) & B(t) \\
C(t) & D(t)
\end{array}\right]
$$

Varía dentro de un polítopo fijo de matrices, lo cual representa la ecuación 10 :

$$
\begin{gathered}
S(t) \in C o\left\{S_{1} \ldots S_{k}\right\}=\sum_{i=1}^{k} \alpha_{i} S_{i}: \alpha_{i} \geq 0, \\
\sum_{i=1}^{k} \alpha_{i}=1
\end{gathered}
$$

Donde $\mathrm{S}_{1} \ldots \mathrm{S}_{\mathrm{k}}$ dan los sistemas vértices, los cuales tienen la forma expresada en la ecuación 11:

$$
S_{i}(t)=\left[\begin{array}{ll}
A(t)_{i} & B(t)_{i} \\
C(t)_{i} & D(t)_{i}
\end{array}\right], i \in[1, k]
$$

En otras palabras, $\mathrm{S}(\mathrm{t})$ es una combinación convexa de sistemas de matrices $\mathrm{S}_{1} \ldots \mathrm{S}_{\mathrm{k}}$. Los números no negativos $\alpha_{1} \ldots \alpha_{\mathrm{k}}$ son llamados coordenadas politópicas de S. La Figura 1 muestra una representación gráfica de la matriz $\mathrm{S}(\mathrm{t})$.

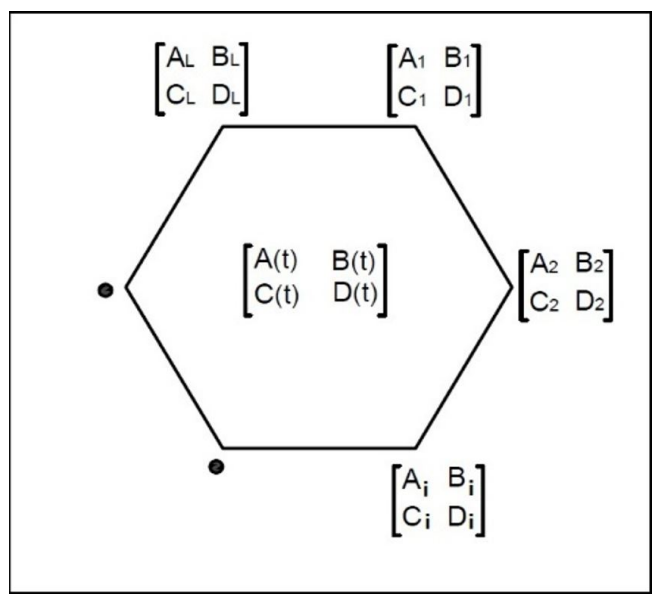

Figura 1. Representación gráfica de la incertidumbre politópica. 


\subsection{Especificación de las regiones de los polos en el plano complejo}

Las regiones LMI, en términos de control, son lugares en los que uno puede lograr ubicar los polos del sistema para determinar sus características de desempeño temporal.

Mediante estas regiones se pueden especificar criterios de desempeño dinámico del sistema como, por ejemplo; el amortiguamiento y el tiempo de respuesta.

Una de estas regiones es la región del semiplano a la izquierda de $-\alpha_{S}$ : Esta región garantiza que el sistema presente un tiempo de respuesta máximo determinado. En la Figura 2 se observa la región donde se desea que los polos se encuentren, que define el área a la izquierda del eje dibujado en $-\alpha_{\mathrm{S}}$ :

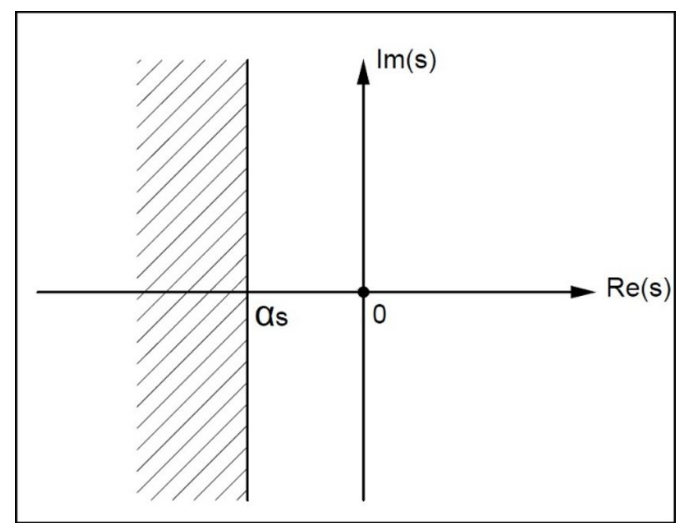

Figura 2. Polos a la izquierda del eje imaginario desplazado $\alpha_{\mathrm{S}}$.

Para lograr esta área debe cumplirse la ecuación 12:

$$
D=\left\{s \in C: s+\bar{s}+2 \alpha_{S}<0\right\}
$$

Donde C representa el plano complejo, D es un subconjunto del plano $\mathrm{C}$, y la condición final es llamada función característica de la región de estabilidad denotada por $\mathrm{f}_{\mathrm{D}}$. Otras regiones LMI se muestran en la Tabla 1. Para la formulación mediante LMI de cada una de estas regiones primero debe notarse que todas ellas pueden ser descritas de forma general mediante la ecuación 13:

$$
D=\{s \in C: P+s Q+s Q+\bar{s} Q T<0\}
$$

Donde $\mathrm{P}$ y $\mathrm{Q}$ son matrices reales tal que $\mathrm{P}^{\mathrm{T}}=\mathrm{P}, \mathrm{y}$ la función representada en la ecuación 14:

$$
f_{D}(s)=P+s Q+\bar{s} Q T
$$

Se conoce como la función característica de la región de estabilidad. El conjunto incluye todas las regiones limitadas descritas anteriormente como sector disco, sector cónico, barras, etc. Mediante intersecciones de regiones se puede obtener virtualmente cualquier forma de región deseada. La intersección de $\mathrm{k}$ regiones viene definida por la ecuación 15 :

$$
P+s Q+\bar{s} Q T<0
$$

Donde $\mathrm{P}$ es una matriz diagonal cuyos elementos no nulos cumplen la condición establecida por la ecuación 16 :

$$
p_{i j}=P_{y}, i=j, j=y, y \in[1, k]
$$

La matriz Q es también una matriz diagonal, cuyos elementos no nulos cumplen con la ecuación 17:

$$
q_{i j}=Q_{y}, i=j, j=y, y \in[1, k]
$$

Donde se identifica la representación global de la región mediante la ecuación 14 .

Tabla 1. Regiones LMI

\begin{tabular}{cc}
\hline Sector & Condición \\
\hline $\begin{array}{c}\text { Semiplano } \\
\text { izquierdo }\end{array}$ & $R(s)<-\alpha_{s} \leftrightarrow s+\bar{s}+a \alpha_{s}<0$ \\
Sector & Sea: $\{s \in C \mid R(s) \tan (\theta)<F(s) ; R(s) \tan (\theta)<-F(s)\}$ \\
cónico & Entonces: $\left(\begin{array}{cc}(s+\bar{s}) \sin (\theta) & (s-\bar{s}) \cos (\theta) \\
(s-\bar{s}) \cos (\theta) & (s+\bar{s}) \sin (\theta)\end{array}\right)<0$ \\
Sector & $|s|<r \leftrightarrow\left(\begin{array}{cc}-r & \bar{s}+q \\
\bar{s}+q & -r\end{array}\right)<0$ \\
disco & Sea: $\left\{s \in C \mid F(s)<\alpha_{s} ;-F(s)<\alpha_{s}\right\} y F(s)=(s-\bar{s})$ \\
$\begin{array}{c}\text { Barras } \\
\text { horizontales }\end{array}$ & Entonces: $\left[\begin{array}{cc}(s-\bar{s})-2 \alpha_{s} & 0 \\
0 & -(s-\bar{s})-2 \alpha_{s}\end{array}\right]<0$ \\
$\begin{array}{c}\text { Sector } \\
\text { disco }\end{array}$ & $\alpha 1_{s}<R(s)<\alpha 2_{s} \leftrightarrow\left(\begin{array}{cc}(s+\bar{s})-2 \alpha 2_{s} & 0 \\
0 & -(s+\bar{s})-2 \alpha 1_{s}\end{array}\right)<0$ \\
\hline
\end{tabular}

\subsection{Formulación de la LMI para ubicación de polos en regiones del plano complejo}

La formulación de la LMI para ubicar los polos en regiones deseadas del plano complejo s se debe a $\mathrm{M}$. Chilali y Gahinet [3]: considérese que $\mathrm{P}^{\mathrm{T}}=\mathrm{P}, \mathrm{Q}$ y A son matrices reales. Entonces A tiene todos sus valores propios en una región estable definida por la ecuación 13, si y solo si existe una matriz real simétrica $\mathrm{X}=\mathrm{X}^{\mathrm{T}}>0$, la cual cumpla con la ecuación 18:

$$
\left(\begin{array}{ccc}
p_{11} X+q_{11} A X+q_{11} X A^{T} & \cdots & p_{1 k} X+q_{1 k} A X+q_{k 1} X A^{T} \\
\vdots & \ddots & \vdots \\
p_{k 1} X+q_{k 1} A X+q_{k 1} X A^{T} & \cdots & p_{k k} X+q_{k k} A X+q_{k k} X A^{T}
\end{array}\right)<0
$$

Donde para este caso $p_{i j}$ y $q_{i j}$ son las i-j ésimas entradas de $\mathrm{P}$ y $\mathrm{Q}$, respectivamente. En otras palabras, A tiene todos sus valores propios en la región de estabilidad D con una función característica $f_{D}$ (s) si y solo si existe una matriz definida positiva $\mathrm{X}$ tal que cumpla con la ecuación 19:

$$
\left(p_{i j} X+q_{i j} X A+q_{i j} A^{T} X\right)<0
$$

Para toda i, j. Nótese que esta es una LMI en X y que el teorema clásico de Lyapunov corresponde a la función característica de la ecuación 20: 


$$
f(s)=s+\bar{s}
$$

Esto equivale entonces a sustituir $(1, \mathrm{~s}, \overline{\mathrm{s}}) \Leftrightarrow\left(\mathrm{A}, \mathrm{AX}, \mathrm{XA}^{\mathrm{T}}\right)[1]$.

\subsection{Normas para señales y sistemas}

Una forma de describir el desempeño de los sistemas de control es en términos del tamaño de ciertas señales de interés. Por ejemplo, el desempeño de un sistema de seguimiento puede ser medido por el tamaño de la señal de error. A continuación se exponen distintas maneras de definir el tamaño de una señal. También se introduce el concepto de normas de funciones de transferencia cuya utilidad radica en que permiten calcular el tamaño de la norma de una señal de salida de un sistema a partir del conocimiento del tamaño de la norma de la señal de entrada.

\subsubsection{Normas para señales}

Una norma debe tener las siguientes propiedades [4], representadas en las ecuaciones 21 a 27 :

$$
\begin{gathered}
\|u\|>0 \\
\|u\|=0 \Leftrightarrow u(t)=0, \forall t \\
\|a u\|=|a|\|u\|, \forall a \in \mathfrak{R} \\
\|u+v\| \leq\|u\|+\|v\|
\end{gathered}
$$

La ecuación 24 es una desigualdad triangular. La ecuación 25 corresponde a una norma 1 de una señal $\mathrm{u}(\mathrm{t})$ :

$$
\|u\|_{1}=\int_{-\infty}^{\infty}|u(t)| d t
$$

La ecuación 26 corresponde a una norma 2 de una señal $u(t)$, esta norma representa la energía de la señal:

$$
\|u\|_{2}=\left(\int_{-\infty}^{\infty} u(t)^{2} d t\right)^{\frac{1}{2}}
$$

En la ecuación 27 se tiene la norma $\infty$ de una señal $\mathrm{u}(\mathrm{t})$, esta norma representa el menor de los límites o cotas superiores del valor absoluto de la señal:

$$
\|u\|_{\infty}=\sup _{t}|u(t)|
$$

\subsubsection{Normas para sistemas}

Se consideran sistemas que son lineales, invariantes en el tiempo y causales. Sea G(s) la función de transferencia del sistema. $\mathrm{G}$ es racional con coeficientes reales. Se dice que $\mathrm{G}$ es estable si no tiene polos en el semiplano cerrado derecho, propia si $\mathrm{G}(\mathrm{j} \infty)$ es finita (grado del denominador $\geq$ grado del numerador), estrictamente propia si $\mathrm{G}(\mathrm{j} \infty)=0$ (grado del denominador $>$ grado del numerador), y bipropia si a la vez $G$ y $G^{-1}$ son propias (grado del numerador $=$ grado del denominador).

Se definen dos normas para la función de transferencia G:

$$
\|G\|_{2}=\left(\frac{1}{2 \pi} \int_{-\infty}^{\infty}\left|G(j w)^{2}\right| d w\right)^{\frac{1}{2}}
$$

La norma 2, ecuación 28, de G es finita si y solo si $\mathrm{G}$ es estrictamente propia y no tiene polos en el eje imaginario. La norma $\|\mathrm{G}\|_{2}$ se convierte en la norma 2 de la señal de salida de un sistema cuando la señal de entrada es una señal impulsiva. También representa la relación $\frac{\|y\|_{\infty}}{\|u\|_{2}}$, que es una relación «pico/energía».

En términos de LMI se tiene que, dada una descripción en espacio de estado, ecuaciones 29-30:

$$
\begin{aligned}
& \dot{x}=A x+B u \\
& y=C x+D u
\end{aligned}
$$

Se sabe que $\mathrm{G}=\mathrm{C}(\mathrm{sI}-\mathrm{A})^{-1} \mathrm{~B}+\mathrm{D}$ y se demuestra en [1] que: $\|\mathrm{G}\|_{2}<\gamma$ si y solo si existen $\mathrm{P}=\mathrm{P}^{\mathrm{T}}>0$ tales que cumplan las ecuaciones $31,32,33$ :

$$
\begin{gathered}
\left(\begin{array}{cc}
A^{T} P+P A & P B \\
B^{T} P & I
\end{array}\right)<0 \\
\left(\begin{array}{cc}
P & C^{T} \\
C & Z
\end{array}\right)<0 \\
\operatorname{trace}(Z)<\gamma^{2}
\end{gathered}
$$

El menor límite superior de la norma $\mathrm{H}_{2}$ de la función de transferencia puede ser calculado minimizando el criterio trace $(\mathrm{Z})$ sobre las variables $\mathrm{K}$ y $\mathrm{Z}$ que satisfacen las LMI definidas por las dos primeras desigualdades.

$$
\|G\|_{\infty}=\sup _{\omega}|G(j \omega)|
$$

La norma $\infty$ de G, ecuación 34, es finita si y solo si $\mathrm{G}$ es propia y no tiene polos en el eje imaginario, además, aparece como el valor pico en el diagrama de Bode de G. La norma $\|\mathrm{G}\|_{\infty}$ se convierte en la norma $\infty$ de la señal de salida de un sistema cuando la entrada es una señal impulsiva.

También representa el valor supremo de la ganancia RMS del sistema, es decir, la relación sup $\left(\frac{\|y\|_{\infty}}{\|u\|_{2}}\right)$. 
En términos de desigualdades matriciales, se tiene según [1] que $\|\mathrm{G}\|_{\infty}<\gamma$ siempre y cuando exista una $\mathrm{P}=\mathrm{P}^{\mathrm{T}}$ que sea solución a la LMI mostrada en la ecuación 35 :

$$
\left(\begin{array}{cc}
A^{T} P+P A+C^{T} C & P B+C^{T} D \\
B^{T} P+D^{T} C & D^{T} D-\gamma^{2} I
\end{array}\right) \leq 0
$$

\section{Síntesis de realimentación de estado}

Se conoce que la técnica de realimentación de estados permite satisfacer especificaciones de estabilidad, desempeño y robustez de los sistemas realimentados. En varios problemas de control, las especificaciones de diseño son una mezcla de objetivos de desempeño y robustez expresados ya sea en el dominio del tiempo o de la frecuencia. Los métodos mediante LMI son bien adaptados a los problemas de síntesis de realimentación de estados con múltiples objetivos [5].

La estructura de control para el diseño de un controlador vía LMI está basado en la realimentación de estados [6], la misma se muestra en la Figura 3:

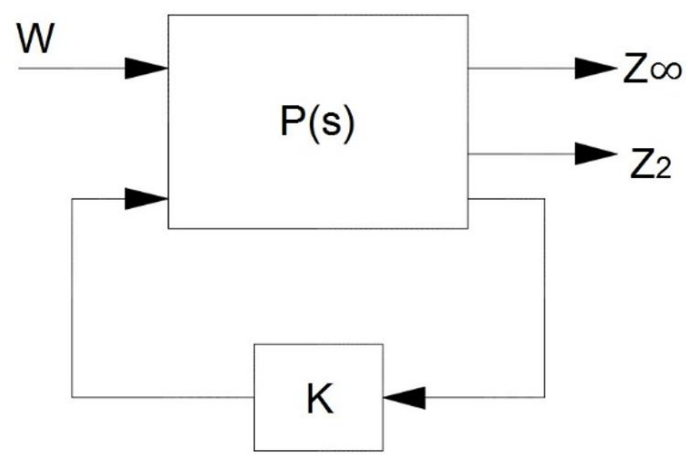

Figura 3. Control por realimentación de estados.

En esta estructura, dada la realización de espacio de estado, ecuaciones 36,37 y 38 :

$$
\begin{gathered}
\dot{x}=A x+B_{1} \omega+B_{2} u \\
z_{\infty}=C_{1} x+D_{11} \omega+D_{12} u \\
z_{2}=C_{2} x+D_{22} u
\end{gathered}
$$

de la planta $\mathrm{P}$, el sistema en lazo cerrado está dado en la forma de espacio de estado por las ecuaciones 39 , 40 y 41 :

$$
\begin{gathered}
\dot{x}=\left(A+B_{2} K\right) x+B_{1} \omega \\
z_{\infty}=\left(C_{1}+D_{12} K\right) x+D_{11} \omega \\
z_{2}=\left(C_{2} x+D_{22} K\right) u
\end{gathered}
$$

Los objetivos de diseño tienen la siguiente formulación LMI:

\subsection{Desempeño $H_{\infty}$}

La ganancia RMS de lazo cerrado desde $\omega$ hacia $Z_{\infty}$ no excede el valor de $\gamma$ si y solo si existe una matriz simétrica $\mathrm{P}$ tal que cumpla la ecuación 42 :

$$
\left(\begin{array}{cc}
\tilde{A}^{T} P+P \tilde{A}+\tilde{C}^{T} \tilde{C} & P \tilde{B}+\tilde{C}^{T} \tilde{D} \\
\tilde{B}^{T} P+\tilde{D}^{T} \tilde{C} & \tilde{D}^{T} \tilde{D}-\gamma^{2} I
\end{array}\right) \leq 0
$$

donde: $\tilde{\mathrm{A}}=\mathrm{A}+\mathrm{B}_{2} \mathrm{~K} ; \tilde{\mathrm{B}}=\mathrm{B}_{1} ; \tilde{\mathrm{C}}=\mathrm{C}_{1}+\mathrm{D}_{12} \mathrm{~K} ;$ $\tilde{\mathrm{D}}=\mathrm{D}_{11}$

\subsection{Desempeño $\mathrm{H}_{2}$}

La norma $\mathrm{H}_{2}$ del lazo cerrado no excede $\gamma$ si existen $\mathrm{P}=\mathrm{P}^{\mathrm{T}}>0$ y $\mathrm{Z}$ tales que cumplan las ecuaciones 43 , 44 y 45 :

$$
\begin{gathered}
\left(\begin{array}{cc}
\left(A+B_{2} K\right)^{T} P+P\left(A+B_{2} K\right) & P B_{1} \\
B_{1}^{T} P & I
\end{array}\right)<0 \\
\left(\begin{array}{cc}
P & \left(C_{2}+D_{22} K\right)^{T} \\
C_{2}+D_{22} K & Z
\end{array}\right)<0 \\
\operatorname{trace}(Z)<\gamma^{2}
\end{gathered}
$$

\subsection{Ubicación de polos en regiones LMI}

El objetivo consiste en encontrar una matriz de realimentación $\mathrm{K}$ de tal forma que permita la ubicación de polos del sistema en lazo cerrado en una región $\mathrm{D}$ del semiplano izquierdo, con el fin de estabilizar el sistema bajo ciertos criterios de desempeño deseados.

Los polos de un sistema en lazo cerrado son ubicados en una región LMI representada por la ecuación 13; Si y solo si existe una matriz simétrica $\mathrm{X}=\mathrm{X}^{\mathrm{T}}$ que satisfaga la ecuación 46 :

$$
\begin{gathered}
{\left[p_{i j} X+q_{i j} X\left(A+B_{2} K\right)+q_{i j}\left(A+B_{2} K\right)^{T} X\right]<0} \\
x>0
\end{gathered}
$$

Si se extiende este criterio para sistemas con incertidumbre, cuyas matrices de espacio varían dentro un polítopo:

$$
\begin{aligned}
& \left(\begin{array}{ll}
A(t)_{1} & B(t)_{1} \\
C(t)_{1} & D(t)_{1}
\end{array}\right) \in \ldots \\
& \cdots \operatorname{Co}\left\{\left(\begin{array}{ll}
A(t)_{k} & B(t)_{k} \\
C(t)_{k} & D(t)_{k}
\end{array}\right): k=1 \ldots K\right\}
\end{aligned}
$$


donde cada modelo politópico es usado para representar cada una de las plantas con incertidumbre, se busca una sola función cuadrática de Lyapunov que permita cumplir con los objetivos de diseño para todas las posibles plantas del polítopo, resultando de esto entonces la siguiente condición LMI:

Los polos de un sistema en lazo cerrado son ubicados en una región LMI como se expresa en la ecuación 13; Si y solo si existe una matriz simétrica $\mathrm{X}=\mathrm{X}^{\mathrm{T}}$ que satisfaga la ecuación 47 :

$$
\begin{gathered}
{\left[p_{i j} X+q_{i j} X\left(A_{k}+B_{2 k} K\right)+q_{i j}\left(A_{k}+B_{2 k} K\right)^{T} X\right]<0} \\
x>0
\end{gathered}
$$

De lo cual, resolviendo la LMI, se obtendrá una solución óptima para $\mathrm{X}$ y una correspondiente matriz de realimentación $\mathrm{K}$ que garantice el cumplimiento de los requerimientos de diseño planteados para cada una de las plantas existentes producto de la incertidumbre dada en el sistema.

\section{Casos de estudio}

\subsection{Caso 1. Sistema doble integrador. Opti- mización de la norma $\mathrm{H}_{2}$}

El problema de equilibrar una bola sobre la superficie de una viga es representativo de una gran clase de sistemas de aplicaciones industriales y militares. El objetivo es controlar la posición de una bola que es libre de moverse a lo largo de una viga rígida sostenida en su punto central. El trabajo del controlador es rotar la viga para compensar el movimiento de la bola. La Figura 4 muestra el esquema de este problema. La posición de la bola es p, el ángulo de la viga es $\theta$, el par de entrada a la viga es $\mathrm{f}$, la inercia de la viga es J y la masa de la bola es $\mathrm{m}$.

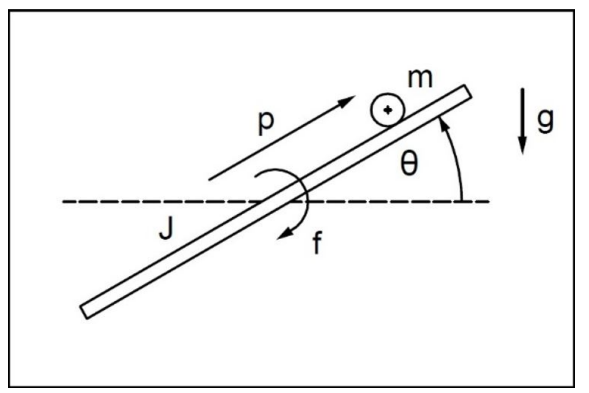

Figura 4. Esquema del problema de equilibrio de una bola en una viga.

Un modelo simplificado de este proceso se observa en la ecuación 48:

$$
\frac{p(s)}{\theta(s)}=K \cdot \frac{1}{s^{2}}
$$

Para el análisis en este proyecto se ha utilizado la función de transferencia normalizada mostrada en la ecuación 49:

$$
P(s)=\frac{1}{s^{2}}
$$

El nombre de sistema doble integrador resulta precisamente de esta última descripción pues como se sabe el término $\mathrm{s}^{-1}$ corresponde en el dominio del tiempo a una integración.

Para este ejemplo se plantea encontrar las ganancias de realimentación de estado que minimicen la norma $\|\mathrm{H}\|_{2}$ de los estados y de la señal de control.

La salida a optimizar se plantea en la forma de la ecuación 50:

$$
z_{2}=\left[\begin{array}{lll}
x_{1} & x_{2} & u
\end{array}\right]^{T}
$$

Se busca, por tanto, minimizar la respuesta transitoria de los estados y de la señal de control en respuesta a entradas conocidas o condiciones iniciales.

Para este caso el problema LMI que resulta es el indicado en la LMI a la cual corresponden las ecuaciones 43,44 y 45 , donde los valores de las matrices se representan en las ecuaciones 51-55:

$$
\begin{gathered}
A=[00 ; 10] \\
B_{1}=[1 ; 0] \\
B_{2}=[1 ; 0] \\
C_{2}=[10 ; 01 ; 00] \\
D_{22}=[0 ; 0 ; 1]
\end{gathered}
$$

La Figura 5 muestra la respuesta impulsiva de los estados y de la señal de control obtenidos.

El valor de ganancia de realimentación resulta ser $\mathrm{K}=[-1.7-1.01]$. La norma mínima $\mathrm{H}_{2}$ que se obtiene es 1.73 .

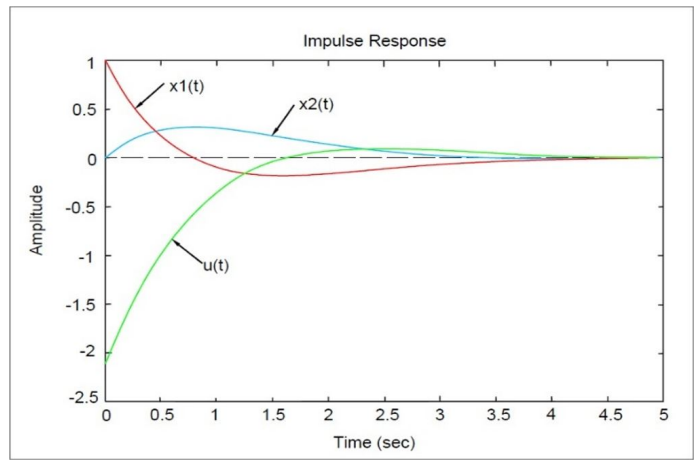

Figura 5. Respuesta impulsiva para sistema doble integrador.

La respuesta muestra que se ha minimizado la energía contenida en el vector z que suma las energías de los estados y la señal de control. La energía es la integral del valor cuadrático de cada variable. 


\subsection{Caso 2. Sistema doble integrador. Sistema de control $\mathbf{H}_{2} / \mathbf{H}_{\infty}$}

En este ejemplo se busca cumplir dos objetivos: primero que el sistema tenga como en el ejemplo anterior una respuesta transitoria adecuada de los estados y de la señal de control y segundo, minimizar el efecto de las perturbaciones en la salida. El segundo objetivo corresponde a minimizar la norma $\mathrm{H}_{\infty}$ de la función de transferencia de perturbación a la entrada $\mathrm{w}(\mathrm{t})$ en la salida $\mathrm{y}(\mathrm{t})$. Es decir,

$$
z_{\infty}=\left[\begin{array}{ll}
0 & 0
\end{array}\right] \cdot x+1 \cdot \omega+0 \cdot u
$$

La ecuación 56 significa resolver las LMI correspondientes a cada problema de desempeño. En la LMI representada por la ecuación 42 , los valores de sus matrices se representan en las ecuaciones 57-61:

$$
\begin{aligned}
& A=[00 ； 10] \\
& B_{1}=[1 ; 0] \\
& C_{1}=\left[\begin{array}{ll}
0 & 1
\end{array}\right] \\
& D_{11}=[0] \\
& D_{12}=[0]
\end{aligned}
$$

En la LMI representada por las ecuaciones 43-45, los valores de sus matrices son ahora los asignados en las ecuaciones $62-66$ :

$$
\begin{gathered}
A=[00 ; 10] \\
B_{2}=[1 ; 0] \\
C_{1}=[10 ; 01 ; 00] \\
D_{21}=[0 ; 0 ; 0] \\
D_{22}=[0 ; 0 ; 1]
\end{gathered}
$$

El proceso [5] inicia encontrando el rango de variación de la norma $\mathrm{H}_{\infty}$. Luego se calcula para un conjunto de normas $\mathrm{H}_{\infty}$ predefinidas, el mejor desempeño posible de la norma $\mathrm{H}_{2}$. Como resultado se obtiene una gráfica como la de la Figura 6 que permite determinar el mejor desempeño deseado.

Por inspección de la Figura 6 se determina que el valor de ganancia $\mathrm{K}$ obtenido para cuando $\mathrm{g}=0.1$ produce un adecuado compromiso de desempeño puesto que en este caso los valores obtenidos son: norma $\mathrm{H}_{2}=3.57$; norma $\mathrm{H}_{\infty}=0.096$. Para este caso se tiene que el valor de $\mathrm{K}=[-4.68-10.45]$ produce las respuestas impulsivas de la Figura 7.

La Figura 7 muestra que la energía contenida en las señales no cambia significativamente, sin embargo, se requiere una señal de control de mayor valor para minimizar el efecto de las perturbaciones en la salida.

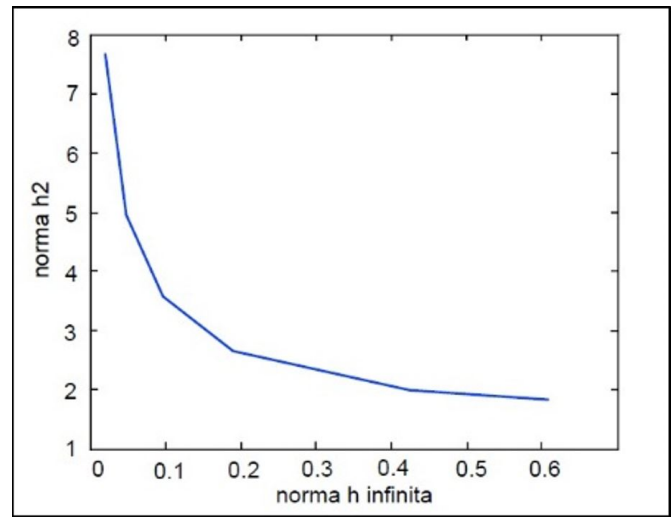

Figura 6. Compromiso entre los desempeños $\mathrm{H}_{2}$ y $\mathrm{H}_{\infty}$.

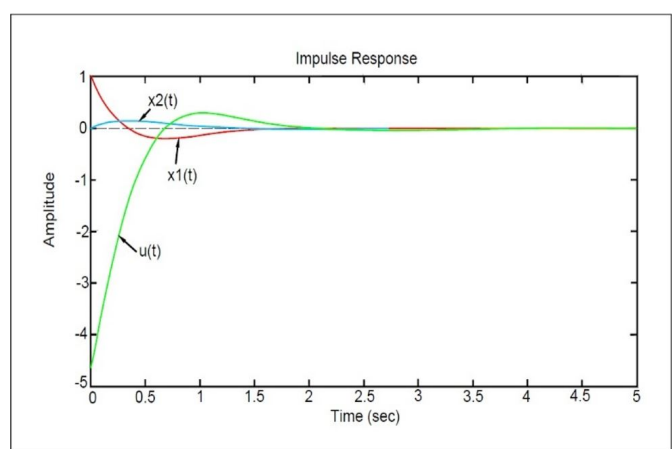

Figura 7. Respuesta impulsiva.

\subsection{Caso 3. Sistema de levitación magnética. Ubicación de polos en regiones LMI para un sistema con incertidumbre paramétrica.}

Para explicar el diseño de un controlador mediante LMI ante la existencia de incertidumbres paramétricas, se usará un sistema de suspensión magnética, en el que una bola de material metálico se suspende mediante un electroimán de corriente controlada por realimentación a través de una medición óptica de la posición de la bola, tal como se muestra en la Figura 8. Este sistema tiene los ingredientes básicos de sistemas para levitación de masas usados en giroscopios, acelerómetros y trenes de alta velocidad [7]. El sistema está representado a través de las ecuaciones 67 y 68 :

$$
\begin{gathered}
M \cdot \frac{d^{2} h}{d t^{2}}=M \cdot g-\frac{K \cdot i^{2}}{h} \\
V=L \cdot \frac{d i}{d t}+R \cdot i
\end{gathered}
$$

Donde h representa la posición vertical de la bola en metros $(\mathrm{m})$, i es la corriente en el electromagneto en amperios (A), $\mathrm{V}$ es la tensión aplicada en voltios $(\mathrm{V}), \mathrm{M}$ es la masa de la bola en kilogramos $(\mathrm{kg})$, g es la aceleración de la gravedad en metros por segundo 
cuadrado $\left(\mathrm{m} / \mathrm{s}^{2}\right)$, L es la inductancia en henrios $(\mathrm{H})$, $\mathrm{R}$ es la resistencia en ohmios $(\Omega)$ y $\mathrm{K}$ es el coeficiente que determina la fuerza magnética ejercida a la bola, adimensional.

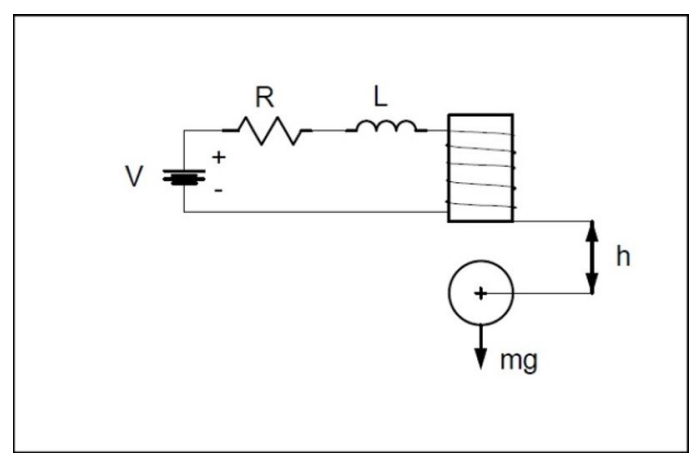

Figura 8. Sistema de suspensión magnética.

Dentro del sistema cabe destacar que se va a considerar incertidumbre en tres parámetros: en M (cuyo valor medido fue de aproximadamente $0,05 \mathrm{~kg}$ ), en $\mathrm{L}$ (cuyo valor modelado de $0,01 \mathrm{H}$ puede variar con respecto al real por error en las fórmulas del modelo como por ejemplo el número exacto de vueltas y las consideraciones que se hacen al tomar un diámetro constante para toda la bobina) y R. Para el resto de parámetros se colocarán los siguientes valores constantes: $\mathrm{K}=0,0001$ y g $=9,81 \mathrm{~m} / \mathrm{seg}^{2}$.

Puesto que el sistema es de tipo no lineal a continuación se procederá a linealizar el sistema. Para ello se dirá que el sistema se encuentra en equilibrio (la bola está suspendida en el aire) siempre que se considere la ecuación 69:

$$
h=\frac{K \cdot i^{2}}{M \cdot g}
$$

Y considerando el punto donde $\frac{d h}{d t}=0$; se linealizarán las ecuaciones alrededor del punto $\mathrm{h}=0,01 \mathrm{~m}$, aplicando la ecuación 70:

$$
i=\sqrt{h \cdot M \cdot g / K}
$$

Con lo cual finalmente se obtiene la siguiente representación en espacio de estados, ecuaciones 71-85:

$$
\begin{gathered}
\dot{x}=A x+B u \\
y=C x+D u \\
x_{1}=h(t) \\
\dot{x}_{1}=\frac{d h(t)}{d t} \\
\dot{x}_{2}=\frac{d^{2} h(t)}{d t^{2}} \\
f_{1}=\dot{x}_{1}=x_{2} \\
f_{2}=\dot{x}_{2}=g-\frac{\frac{0.0001}{M}}{x_{1}} \cdot x_{3}^{2}
\end{gathered}
$$

$$
f_{3}=\dot{x}_{3}=\frac{V}{L}-\frac{R}{L} \cdot x_{3}
$$

Condiciones iniciales, ecuaciones 79-81:

$$
\begin{gathered}
x_{1}=h_{0}=0.01 m \\
x_{2}=\frac{d h}{d t}=0 \\
x_{3}=\sqrt{h_{0} \cdot M \cdot g / K} \\
A=\left[\begin{array}{lll}
\frac{\partial f_{1}}{\partial x_{1}} & \frac{\partial f_{1}}{\partial x_{2}} & \frac{\partial f_{1}}{\partial x_{3}} \\
\frac{\partial f_{2}}{\partial x_{1}} & \frac{\partial f_{2}}{\partial x_{2}} & \frac{\partial f_{2}}{\partial x_{3}} \\
\frac{\partial f_{3}}{\partial x_{1}} & \frac{\partial f_{3}}{\partial x_{2}} & \frac{\partial f_{3}}{\partial x_{3}}
\end{array}\right]=\left[\begin{array}{ccc}
0 & 1 & 0 \\
980 & 1 & \frac{-0.626}{\sqrt{M}} \\
0 & 1 & \frac{-R}{L}
\end{array}\right] \\
B=\left[\begin{array}{c}
\frac{\partial f_{1}}{\partial V} \\
\frac{\partial f_{2}}{\partial V} \\
\frac{\partial f_{3}}{\partial V}
\end{array}\right]=\left[\begin{array}{cc}
0 \\
0 \\
\frac{1}{L}
\end{array}\right]= \\
\left.\frac{\partial Y}{\partial x_{2}} \quad \frac{\partial Y}{\partial x_{3}}\right]=\left[\begin{array}{lll}
1 & 0 & 0
\end{array}\right] \\
D=\left[\begin{array}{ll}
\frac{\partial Y}{\partial x_{1}} \\
C=
\end{array}\right]
\end{gathered}
$$

Donde $\mathrm{x}=\left[\mathrm{x}_{1} ; \mathrm{x}_{2} ; \mathrm{x}_{3}\right]$ es el conjunto de variables de estado del sistema, u es la tensión de entrada $\mathrm{V}$ y la salida Y es la altura h.

A partir de este momento entonces se procede a diseñar el controlador vía LMI a través de criterios tales como la estabilidad y ubicación de polos teniendo en cuenta la incertidumbre del sistema. En primer lugar, se procede a analizar el sistema ideal sin incertidumbre (con los valores nominales de los parámetros: $\mathrm{M}=0,05$ $\mathrm{kg}, \mathrm{L}=0,01 \mathrm{H}$ y $\mathrm{R}=1 \Omega$ ) para ver si este es estable o inestable. Las matrices correspondientes para este sistema se representan en las ecuaciones 86-88:

$$
\begin{gathered}
A=\left[\begin{array}{ccc}
0 & 1 & 0 \\
980 & 1 & -2.8 \\
0 & 0 & -100
\end{array}\right] \\
B=\left[\begin{array}{c}
0 \\
0 \\
100
\end{array}\right] \\
C=\left[\begin{array}{lll}
1 & 0 & 0
\end{array}\right]
\end{gathered}
$$

Los polos del sistema se hallan a través de los valores propios de matriz $\mathrm{A}$, sus valores se muestran en la Tabla 2:

Tabla 2. Polos del sistema de levitación magnética

\begin{tabular}{cc}
\hline Polo 1 & 31,3050 \\
Polo 2 & $-31,3050$ \\
Polo 3 & -100 \\
\hline
\end{tabular}

Como se puede apreciar, uno de los polos se halla en el semiplano derecho, lo que significa que el sistema es inestable en lazo abierto. Se puede entonces construir un controlador en especial, de los que presenta la teoría de control clásica, cuyos criterios de diseño 
sean un tiempo de establecimiento ts $<0,5$ seg y un sobrepico $\mathrm{Mp}<5 \%$. Lamentablemente al tomar en cuenta la existencia de incertidumbre en el sistema, los controladores clásicos ya no pueden cumplir con los criterios de desempeño deseados; ya que su uso está limitado única y exclusivamente para sistemas sin incertidumbre.

Es por esta razón por la que se diseñará un controlador vía LMI que permita cumplir con los requerimientos del sistema tomando en cuenta la incertidumbre dada en el sistema.

El diseño a implementarse utilizará la ley de realimentación de estados más control integral que presenta la estructura mostrada en la Figura 9:

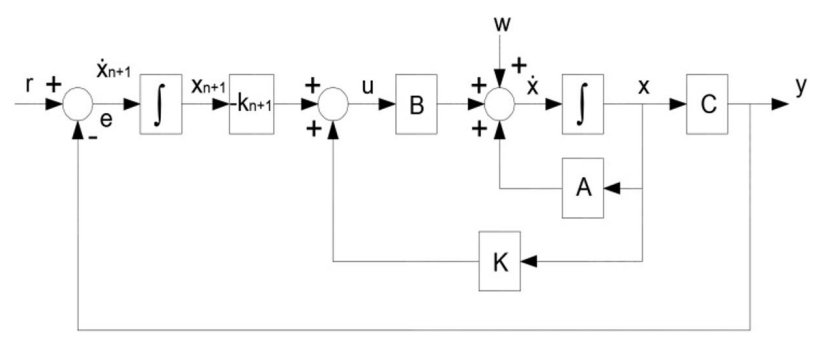

Figura 9. Control por realimentación de estados más control integral.

Este esquema tiene la ventaja de poder garantizar una condición de desempeño ante perturbaciones estacionarias, en la cual el sistema puede realizar un seguimiento de la señal de referencia sin error lo que representa una condición más interesante que la que se obtiene por realimentación simple de estados [8]. El sistema linealizado a lazo cerrado para este esquema queda definido por las siguientes ecuaciones diferenciales, ecuaciones 89-90:

$$
\begin{gathered}
\dot{\bar{x}}=(\bar{A}+\overline{B K}) \cdot \bar{x}+\left[\begin{array}{l}
0 \\
1
\end{array}\right] \cdot r \\
y=C x+D u
\end{gathered}
$$

En donde el vector de estados y las matrices del sistema a lazo cerrado ahora pasan a ser como se muestra en las ecuaciones 91-93:

$$
\begin{gathered}
\bar{x}=\left[\begin{array}{c}
x \\
x_{n-1}
\end{array}\right] \\
\bar{A}=\left[\begin{array}{cc}
A & 0 \\
-C & 0
\end{array}\right] \\
\bar{B}=\left[\begin{array}{c}
B \\
0
\end{array}\right]
\end{gathered}
$$

En el nuevo vector de estados del sistema $\overline{\mathrm{x}}$, se observan los estados originales de la planta y un nuevo estado que resulta de la incorporación de un integrador asociado a la señal de error resultante de la diferencia entre la referencia y el valor de la salida del sistema, con lo que se pretende asegurar el seguimiento de la señal de referencia que permita eliminar el error del sistema ante valores constantes de la misma.

El nuevo vector de ganancias de realimentación estaría dado por la ecuación 94:

$$
\bar{K}=\left[\begin{array}{ll}
K & k_{n+1}
\end{array}\right]
$$

Donde $\mathrm{k}_{\mathrm{n}+1}$ será la ganancia correspondiente para la parte integral.

La ley de control estaría descrita por la ecuación 95:

$$
u=K x+k_{n+1} x
$$

Finalmente, las matrices para la nueva representación en espacio de estados quedarían expresadas de la siguiente manera conforme las ecuaciones 96-99:

$$
\begin{gathered}
\bar{B}=\left[\begin{array}{l}
0 \\
0 \\
\frac{1}{L} \\
0
\end{array}\right] \\
C=\left[\begin{array}{lll}
1 & 0 & 0
\end{array}\right] \\
D=\left[\begin{array}{ll}
0
\end{array}\right]
\end{gathered}
$$$$
\bar{A}=\left[\begin{array}{cc}
A & 0 \\
-C & 0
\end{array}\right]=\left[\begin{array}{cccc}
0 & 1 & 0 & 0 \\
980 & 1 & \frac{-0.626}{\sqrt{M}} & 0 \\
0 & 1 & \frac{-R}{L} & 0 \\
-1 & 0 & 0 & 0
\end{array}\right]
$$

Con esta representación, a continuación, se procede a formar la LMI que dará solución al sistema. Primero se introducirá la incertidumbre en el sistema y por facilidad se lo hará a través del modelo politópico, el cual por motivo de la incertidumbre que presentan la masa $\mathrm{M}$, la inductancia $\mathrm{L}$ y la resistencia $\mathrm{R}$ en el rango:

$$
M \in[0.04,0.06] ; L \in[0.008,0.012] ; R \in[0.8,1.2]
$$

Se caracterizará por poseer 8 posibles sistemas extremos como muestra la Tabla 3.

En segundo lugar, ubicamos la región LMI en donde se ubicarán los polos del sistema en lazo cerrado y que permita cumplir con los requerimientos del sistema deseados. Una región adecuada para este caso corresponde a la intersección de las regiones: semiplano izquierdo y sector disco, con las cuales se asegura la rapidez del sistema junto a un limitado máximo pico.

Las matrices $\mathrm{P}$ y $\mathrm{Q}$ formadas a raíz de la intersección de las dos regiones anteriores, ecuación 13, son las siguientes:

Región semiplano izquierdo: de la forma:

$\mathrm{s}+\overline{\mathrm{s}}+2 \alpha_{\mathrm{s}}$ y definida por las ecuaciones $100-102$ :

$$
P_{1}=\left[2 \alpha_{s}\right]
$$




$$
\begin{aligned}
Q_{1} & =[1] \\
Q_{1}^{T} & =[1]
\end{aligned}
$$

Tabla 3. Matrices vértices del sistema politópico

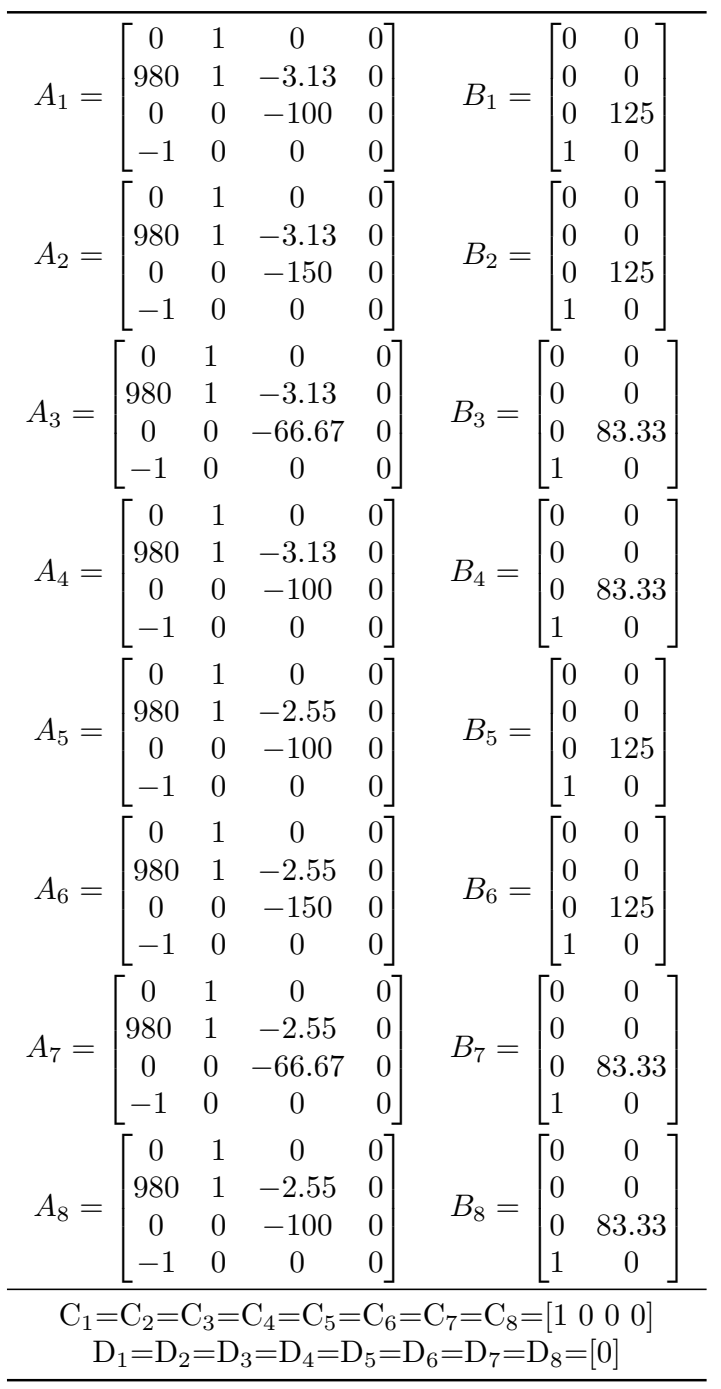

Sector disco: que tiene la forma matricial $\left(\begin{array}{cc}-r & \bar{s}+q \\ s+q & -r\end{array}\right)$ y está definida por 103-105:

$$
\begin{gathered}
P_{2}=\left[\begin{array}{cc}
-r & q \\
q & -r
\end{array}\right] \\
Q_{2}=\left[\begin{array}{ll}
0 & 0 \\
1 & 0
\end{array}\right] \\
Q_{2}^{T}=\left[\begin{array}{ll}
0 & 1 \\
0 & 0
\end{array}\right]
\end{gathered}
$$

Intersección: que está definida por las ecuaciones 106 y 107 :

$$
P=\left[\begin{array}{cc}
P_{1} & 0 \\
0 & P_{2}
\end{array}\right]=\left[\begin{array}{ccc}
2 \alpha_{s} & 0 & 0 \\
0 & -r & q \\
0 & q & -r
\end{array}\right]
$$

$$
Q=\left[\begin{array}{cc}
Q_{1} & 0 \\
0 & Q_{2}
\end{array}\right]=\left[\begin{array}{lll}
1 & 0 & 0 \\
0 & 1 & 0 \\
0 & 0 & 1
\end{array}\right]
$$

Por último, pasamos a formular las LMI de las cuales se obtendrá la matriz de Lyapunov X que permitirá cumplir con el criterio de estabilidad, así como también la matriz de retroalimentación $\mathrm{K}$.

La condición LMI será: Los polos del sistema en lazo cerrado son ubicados en la región LMI representada por la ecuación 13; Si y solo si existe una matriz simétrica de la forma:

$$
\mathrm{X}=\mathrm{X}_{1}=\mathrm{X}_{2}=\mathrm{X}_{3}=\mathrm{X}_{4}=\mathrm{X}_{5}=\mathrm{X}_{6}=\mathrm{X}_{7}=\mathrm{X}_{8}=\mathrm{X}^{\mathrm{T}}
$$

Con:

$$
\mathrm{Y}=\mathrm{Y}_{1}=\mathrm{Y}_{2}=\mathrm{Y}_{3}=\mathrm{Y}_{4}=\mathrm{Y}_{5}=\mathrm{Y}_{6}=\mathrm{Y}_{7}=\mathrm{Y}_{8}=\mathrm{K} \cdot \mathrm{X}
$$

Que satisfaga las ecuaciones con la forma general presentada en la ecuación 108:

$$
\left[\begin{array}{ccc}
E 1 X_{i} & 0 & 0 \\
0 & -r X_{i} & E 3 \\
0 & E 2 & -r X_{i}
\end{array}\right]<0
$$

Dónde las matrices E1, E2 y E3 están representadas por las ecuaciones 109-111:

$$
\begin{gathered}
E 1=\left(2 \alpha_{s}+A_{i}+A_{i}^{T}\right) \cdot X_{i} \\
E 2=\left(q+A_{i}\right) X_{i}+B_{u i} Y_{i} \\
E 3=\left(q+A_{i}^{T}\right) X_{i}+\left(B_{u i} Y_{i}\right)^{T}
\end{gathered}
$$

Teniendo en cuenta que: $\mathrm{i} \in[1,8] \mathrm{y}$, además, $\mathrm{X}>0$.

Para dar solución a todas estas LMI de una manera más rápida y eficiente, se usó el software LMI Control Toolbox de Matlab; mediante el cual se llegó a definir la región intersectada por: semiplano izquierdo: con punto inicial $\alpha_{\mathrm{s}}=-10$ y sector disco: con centro $\mathrm{q}=0$ y radio $\mathrm{r}=126$, como las condiciones más adecuadas para cumplir con los requerimientos deseados del sistema.

\section{Resultados y discusión}

Definido entonces todo el sistema de LMI, las matrices de retroalimentación $\mathrm{K}$ y de Lyapunov X arrojadas se representan en las ecuaciones 112 y 113 respectivamente:

$$
K=\left[\begin{array}{llll}
1132 & 30 & -0.2 & -6064
\end{array}\right]
$$




$$
X=\left[\begin{array}{cccc}
0.0934 & -2.9201 & 0.102 & 0.003 \\
-2.920 & 91.6969 & 3.826 & -0.0930 \\
0.102 & 3.8257 & 251.407 & 0.0052 \\
0.003 & -0.0930 & 0.00520 & 0.0001
\end{array}\right]
$$

Siendo la ecuación 114 la ganancia correspondiente para el factor integral.

$$
k_{n+1}=K(1,4)=-6064
$$

Para evaluar el comportamiento del sistema con incertidumbre en general se grafica la respuesta temporal de todos los sistemas matrices vértices que conforman el sistema politópico producto de la incertidumbre ante una entrada escalón de 0.01 (altura deseada de la bola en metros) bajo condiciones iniciales no nulas $(\mathrm{h}=0.005 \mathrm{~m} ;(\mathrm{dh}) / \mathrm{dt}=0 ; \mathrm{i}=0 \mathrm{~A})$. Estas respuestas obtenidas se presentan en la Figura 10:

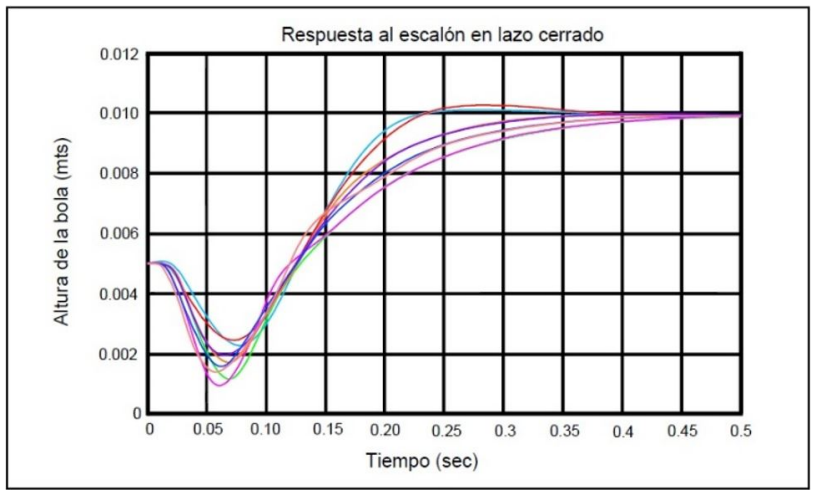

Figura 10. Respuestas del sistema de levitación en lazo cerrado con incertidumbre.

Se aprecia con esto claramente como la distancia entre la bola y el electromagneto sigue fielmente a la señal de referencia (distancia deseada), con las características de requerimiento deseado (ts $<0,5(\mathrm{~s})$ y $\mathrm{Mp}<5 \%$ ).

\section{Conclusiones}

El método de diseño de sistemas de control robustos mediante realimentación de estados utilizando la técnica de las desigualdades lineales matriciales (LMI) demuestra ser atractivo para problemas de control en los cuales se especifican múltiples objetivos entre los que destacan la ubicación específica de polos del sistema y valores deseados de los índices de desempeño $\mathrm{H}_{2}$ y $\mathrm{H}_{\infty}$ que se plantean dentro de la estructura del sistema de control a diseñarse.

La técnica de diseño mediante LMI permite resolver problemas de rechazo a perturbaciones mediante la optimización de la norma $\mathrm{H}_{\infty}$ de la función de transferencia desde la perturbación a la salida, así como también limitar la energía de los estados y de la señal de control mediante la optimización de la norma $\mathrm{H}_{2}$ del vector formado por estas señales.

El uso de LMI en problemas de diseño de controladores tiene como ventaja no solo el de satisfacer condiciones mínimas de desempeño dinámico tales como la estabilidad, sino que también puede asegurar otro tipo de especificaciones puntuales. Por ejemplo. se puede elegir ubicar los polos de un sistema en regiones LMI tales como sector disco, sector cónico, semiplano izquierdo, etc., a fin de conseguir valores específicos de coeficiente de amortiguamiento, frecuencia natural no amortiguada, tasa de decaimiento de la señal, entre otros, que marcan los parámetros de desempeño de respuesta transitoria que se desean alcanzar. Así mismo se tiene la posibilidad de diseñar controladores con normas $\mathrm{H}_{2}$ y $\mathrm{H}_{\infty}$ que permitan reducir la ganancia de perturbación dada en un sistema prediciendo además hasta qué punto máximo de perturbación puede soportar el mismo.

Con base en los casos de estudio aquí presentados, el diseño de controladores por LMI en combinación con la teoría de espacio de estados, al tener como fundamento una matemática del tipo matricial, se presenta como alternativa para ser aplicada a sistemas prácticos del tipo no lineal, al limitar estos a una zona de trabajo lineal específica, con incertidumbre paramétrica adjunto a perturbaciones con resultados ventajosos y que no se los puede conseguir simplemente con el uso de la teoría de control clásica cuyos controladores, en la mayoría de casos, carecen de robustez.

\section{Referencias}

[1] C. Scherer and S. Weiland, "Lecture notes DISC course on linear matrix inequalities in control," 1999. [Online]. Available: https://goo.gl/gVHP91

[2] J. P. Hespanha, "Undergraduate lecture notes on robust control," in University of California, Santa Barbara, 2007, pp. 1-23. [Online]. Available: https://goo.gl/a5igpY

[3] M. Chilali, P. Gahinet, and P. Apkarian, "Robust pole placement in lmi regions," IEEE Transactions on Automatic Control, vol. 44, no. 12, pp. 2257-2270, Dec 1995. [Online]. Available: https://doi.org/10.1109/9.811208

[4] J. Doyle, B. Francis, and A. Tannenbaum, "Feedback control theory," in Macmillan Publishing Co., 1990. [Online]. Available: https://goo.gl/gJ39NF

[5] P. Gahinet, A. Nemirovski, A. J. Laub, and M. Chilali, "LMI control toolbox for use with MATLAB," in The MathWorks, 1995. [Online]. Available: https://goo.gl/K4nCuH 
[6] J. Cotero, "Control clásico/moderno y control inteligente," 2005. [Online]. Available: https://goo.gl/6LYGbb

[7] B. Messner and D. Tilbur. Tutorial de espacio de estados. [Online]. Available: https://goo.gl/Atp3CN
[8] O. Pérez, P. Vega, W. Colmenares, and M. Francisco, "Diseño integrado con realimentación robusta de estados vía desigualdades lineales matriciales," IEEE Latin America Transactions, vol. 2, no. 3, pp. 174-182, 2004. [Online]. Available: https://goo.gl/WFyB2w 